\title{
Transforming growth factor-beta signaling network regulates plasticity and lineage commitment of lung cancer cells
}

\author{
I Ischenko', J Liu, O Petrenko² and MJ Hayman ${ }^{\star, 1}$
}

Identification of target cells in lung tumorigenesis and characterization of the signals that control their behavior is an important step toward improving early cancer diagnosis and predicting tumor behavior. We identified a population of cells in the adult lung that bear the EpCAM + CD104 + CD49f + CD44 + CD24loSCA1 + phenotype and can be clonally expanded in culture, consistent with the properties of early progenitor cells. We show that these cells, rather than being restricted to one tumor type, can give rise to several different types of cancer, including adenocarcinoma and squamous cell carcinoma. We further demonstrate that these cells can be converted from one cancer type to the other, and this plasticity is determined by their responsiveness to transforming growth factor (TGF)-beta signaling. Our data establish a mechanistic link between TGF-beta signaling and SOX2 expression, and identify the TGF-beta/SMAD/SOX2 signaling network as a key regulator of lineage commitment and differentiation of lung cancer cells.

Cell Death and Differentiation (2014) 21, 1218-1228; doi:10.1038/cdd.2014.38; published online 28 March 2014

Lung cancer is the leading cause of cancer-related mortality in both men and women worldwide. Lung cancers are divided into two major categories: non-small-cell lung cancer (NSCLC) and small-cell lung cancer. NSCLC accounts for $\sim 80 \%$ of all lung cancers and is divided further into adenocarcinoma (ADC), squamous cell carcinoma (SCC) and large-cell lung carcinoma. Of the four major types of lung cancer, Kras mutations are present in about $30-50 \%$ of ADC, a smaller percentage of SCC (5-7\%) and $<1 \%$ of SCLC. ${ }^{1,2}$ Mutations of the p53 gene are common in all types of lung cancer and range from $\sim 30 \%$ in ADC to more than $70 \%$ in SCC and SCLC. ${ }^{3}$ Other alterations occur at lower frequencies in NSCLC, including mutations in EGFR (15\%), EML4-ALK (4\%), ERBB2 (2\%), AKT1, BRAF, MAP2K1 and MET.2,4 Previous efforts in comprehensive characterization of lung cancer include copy number and gene expression profiling, targeted sequencing of candidate genes and large-scale genome sequencing of tumor samples. ${ }^{5-9}$ Significant progress has also been made in developing mouse models of lung carcinogenesis. ${ }^{10,11}$ The unifying theme underlying these studies is that there exists a permissive cellular context for each specific oncogenic lesion, and that only certain types of cells are capable of cancer initiation. ${ }^{12-14}$

The lung consists of three anatomically distinct regions such as trachea, bronchioles and alveoli, each maintained by a distinct population of progenitor cells, that is, basal, Clara and alveolar type 2 (AT2) cells, respectively. ${ }^{15,16}$ Previous work has focused upon AT2 cells, Clara cells (or variant Clara cells with low CC10 expression) and the putative bronchioalveolar stem cells (BASCs) as potential cells of origin for lung ADC. ${ }^{12,14,17}$ However, to date, only AT2 cells have been conclusively identified as having the potential to be the cells of origin for lung ADC. ${ }^{14,17}$ This raises the question of whether Clara cells, their restricted subpopulations or the newly identified candidate stem cells, termed distal airway stem cells, ${ }^{18}$ alveolar epithelial progenitor cells (AECs) ${ }^{19,20}$ and BASCs, ${ }^{12}$ also have the capacity to give rise to ADC. Current knowledge on the cellular origins of SCC, the second most common type of lung cancer, lags behind that of ADC, partly owing to the fact that squamous cells are not normally present in the respiratory epithelium, and therefore arise through either metaplasia (conversions between stem cell states) or trans-differentiation (conversions between differentiated cells). ${ }^{21,22}$ Whether the mechanisms of SCC causation vary by cell type, their responses to various cells signaling cascades (e.g., transforming growth factor (TGF)-beta, WNT, etc.), or other tumor characteristics is unknown at present.

To address the questions of cell type of origin and signal cascades that control their behavior, we developed in vitro culture conditions that favor the growth of lung epithelial cells with stem cell-like properties. We describe a population of cells isolated from the adult lung that, rather than being restricted to one tumor type, can give rise to several different types of cancer, including ADC and SCC. We also show that these cells can be converted from one cancer type to the other, and this plasticity is largely, if not solely, determined by TGF-beta signaling.

${ }^{1}$ Department of Molecular Genetics and Microbiology, Stony Brook University, Stony Brook, NY, USA and ${ }^{2}$ Department of Pathology, Stony Brook University, Stony Brook, NY, USA

*Corresponding author: MJ Hayman, Department of Molecular Genetics and Microbiology, Stony Brook University, Stony Brook, NY 11794, USA. Tel: +1 631 632 8792; Fax: +1 631632 8891; E-mail: michael.hayman@stonybrook.edu

Abbreviations: NSCLC, non-small-cell lung cancer; ADC, adenocarcinoma; SCC, squamous cell carcinoma; FACS, fluorescence-activated cell sorting; EpCAM, epithelial cell adhesion molecule; SCA1, stem cell antigen 1; KRT15, keratin-15

Received 21.10.13; revised 24.2.14; accepted 25.2.14; Edited by R De Maria; published online 28.3.14 


\section{Results}

Identification of tumorigenic cells in KrasG12D-induced lung carcinoma. The cell(s) of origin of NSCLC has not been formally identified, although AT2 cells and the putative BASCs have been proposed as initiating cell sources for lung ADC. ${ }^{12,14,17}$ Here, we modeled the development of NSCLC using primary cells derived from crosses of LSL KrasG12D mice with p53-null mice (referred to as LSL KrasG12D $\mathrm{p} 53^{\mathrm{KO}}$ ). These mice allow cre-mediated expression of activated KrasG12D allele at a physiological level. ${ }^{11}$ Lung epithelial cells were isolated by flow cytometry based on their expression of cell surface markers EpCAM, CD104 and SCA1, and lack of expression of CD31, CD45 and/or CD90. ${ }^{20}$ FACS (fluorescence-activated cell sorting) analysis of the purified LSL KrasG12D $p 53^{\mathrm{KO}}$ lung cells showed a nearly uniform expression of stem/progenitor cell markers EpCAM, CD24, CD44, CD49f, CD104 and SCA1 (Figure 1a), with more mature EpCAM-/loCD49f-/loSCA1-/lo cells ${ }^{20}$ representing a minority (Supplementary Figure 1). BASC cells are defined by the surface phenotype of EpCAM + $\mathrm{SCA} 1+\mathrm{CD} 34+$ and expression of both SpC (AT2 cell marker) and CC10 (Clara cell marker). ${ }^{12,14,17}$ Approximately $5 \%$ of our LSL KrasG12D p53 ${ }^{\mathrm{KO}}$ cells were also positive in CD34 staining (Supplementary Figure 2). However, we found virtually no $\mathrm{CC} 10+$ or $\mathrm{SpC}+$ cells, implying that our cultures were not enriched in BASC-like cells. Hence, based on their FACS profile, our LSL KrasG12D $\mathrm{p} 53^{\mathrm{KO}}$ cells are relatively uniform and closely resemble the population of self-renewing EpCAM + CD104 + CD49f + CD24lo AECs of the bronchioalveolar junction regions (Supplementary Figure 1). These cells comprise $\sim 10 \%$ of all lung cells, express little or no $\mathrm{CC} 10$ and $\mathrm{SpC}$, but they are able to give rise to the differentiated airway and alveolar cells. ${ }^{19,20}$

To delete the transcriptional termination cassette (Lox-StopLox) and thus activate the expression of the KrasG12D protein, we used a self-excising retroviral vector expressing Cre recombinase. ${ }^{23}$ The presence of the activated KrasG12D allele was confirmed by western blot analysis of KrasG12D and total Kras expression (Figure 1b), and by detection of active GTPbound Kras protein (Figure 1c). Genomic PCR confirmed the presence of the recombined KrasG12D allele (Figure 1d). FACS analysis revealed that KrasG12D p53 ${ }^{\mathrm{KO}}$ cells retained the major immunophenotypic features of their parental cells (Figure 1a). Gating on the EpCAMhiCD49f + cell fraction (presumptive lung epithelial precursor cells) ${ }^{20}$ showed a substantial increase in the percentage of CD104hiCD44hiCD24lo cells (Figure 1a), but no change in the percentage of $\mathrm{EpCAM}+\mathrm{CD} 34+$ cells (Supplementary Figure 2).

To ascertain the tumorigenic potential of our cell populations, unsorted or FACS-sorted EpCAM + SCA1 + $\mathrm{CD} 34+$ and EpCAM + SCA1 + CD34 - KrasG12D p53 ${ }^{\mathrm{KO}}$ cells were injected subcutaneously into nude mice. We found that FACS-sorted CD34+ and CD34- cells exhibited similar tumorigenicity in vivo (Supplementary Figure 2). Furthermore, in both groups (i.e., CD34+ and CD34 - ), morphologically identifiable tumor types included ADC (resembling human acinar type ADC), SCC and SC (sarcomatoid carcinoma, highly malignant variant of NSCLC) (Figure 1e and Supplementary Figure 3). These data are consistent with previous reports showing that putative BASCs are not the only cell type that gives rise to KrasG12D-driven tumors. ${ }^{14,17}$ More importantly, they demonstrate that our cultures contain cells with the potential to give rise to at least three different types of lung cancers, despite their apparent genetic and immunophenotypic similarity.

Cancers with distinct histological features originate from clonally derived cell populations. To exclude the possibility that the different forms of cancers could result from the expansion of rare KrasG12D p53 ${ }^{\mathrm{KO}}$ cells harboring other positively selected mutations, we generated clonal cell populations. FACS analysis revealed no significant immunophenotypic differences in clonally derived cell lines (Supplementary Figure 2). Therefore, we used established lineage-specific intracellular markers (namely, p63, SOX2 and KRT15) of lung stem/progenitor cells. ${ }^{18,24,25}$ We found the differential expression of these genes, which was maintained in the clonal populations after multiple passages (Figures $2 \mathrm{a}$ and b). Clonal cells were accordingly categorized into three distinct groups: (1) p63 + SOX2-KRT15 - (single positive, SP); (2) p63 + SOX2 + KRT15 - (double positive, $\mathrm{DP}$ ); and (3) p63 + SOX2 + KRT15 + (triple positive, TP) (Figures $2 \mathrm{a}$ and $\mathrm{b}$ ). Although the clonal cell lines displayed distinctive phenotypes, we found no differences in the levels of KrasG12D expression or ERK1/2 phosphorylation (Figure 2c). However, they exhibited markedly different tumorigenic phenotypes (Figure 2d), and generated several distinct types of cancer, rather than being restricted to one type, as judged by histology and marker analysis (KRT15 and p63) (Figure 2e). Thus, when injected into mice, individual SP clones gave rise to two tumor types, ADC and SC; individual DP clones produced ADC, SCC and SC; and the TP clones gave rise to SCC and SC (Supplementary Figure 4). Some of these clonally derived tumors were composed of histologically different components, that is SCC, ADC and/or SC (Supplementary Figure 4), suggesting that they originated from cells capable of multidirectional differentiation. The stem cell-like characteristics of individual cell clones were confirmed based upon their ability to form multicellular spheroids (tumor spheres) in nonadherent serum-free conditions (Supplementary Figure 5). All of the tumor sphere cell populations assayed demonstrated a high level of phenotypic plasticity that enabled them to undergo reversible differentiation and renewed proliferation (Supplementary Figure 5).

TGF-beta-mediated response regulates lineage commitment of lung cancer cells. Loss of the type 2 TGF-beta receptor (TBR2) expression was seen in $>50 \%$ of the tumors in nude mice induced by KrasG12D p53 ${ }^{\mathrm{KO}}$ cells (Figures $3 a$ and $b$ ). All of these TBR2-negative tumors were morphologically pure SCCs and expressed high levels of KRT15 and the transcription factors SKI, p63 and SOX2 (Figures $3 a$ and $b$ ), which are known prognostic indicators and markers of SCC. ${ }^{26-28}$ In contrast, the TBR2-positive tumors were either ADCs or SCs, which had low or no expression of SKI, p63, SOX2 or KRT15 (Figure 3b). To determine whether the loss of TBR2 expression indicated that the TGF-beta signaling had a mechanistic role in the 
a
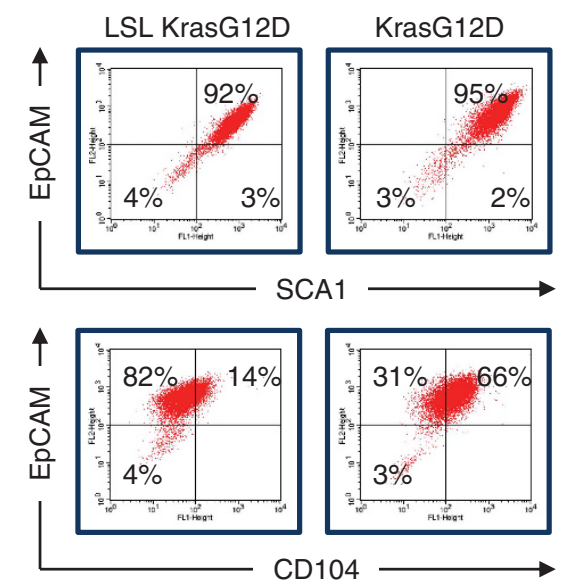

b

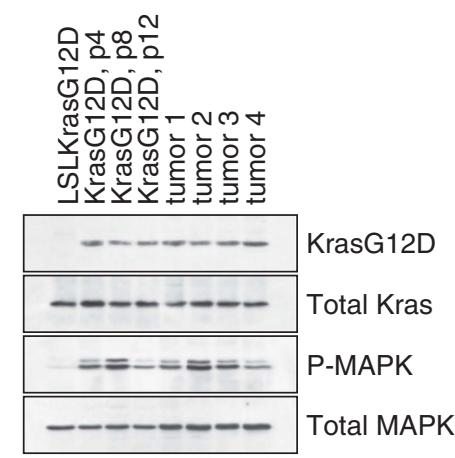

e

e $\quad A D C$
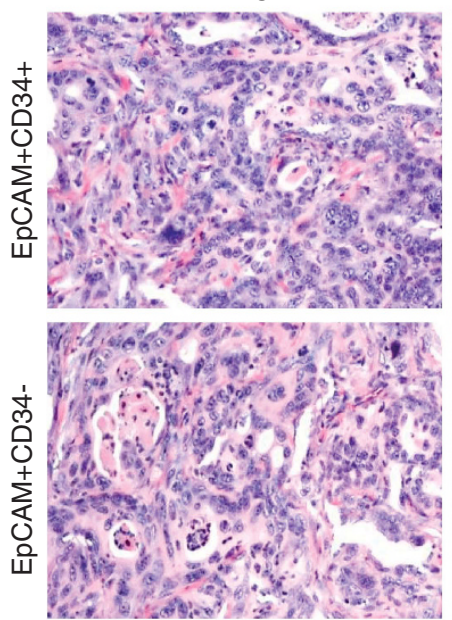

c

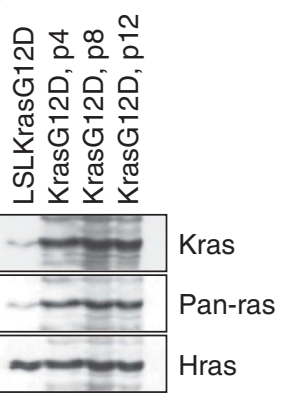

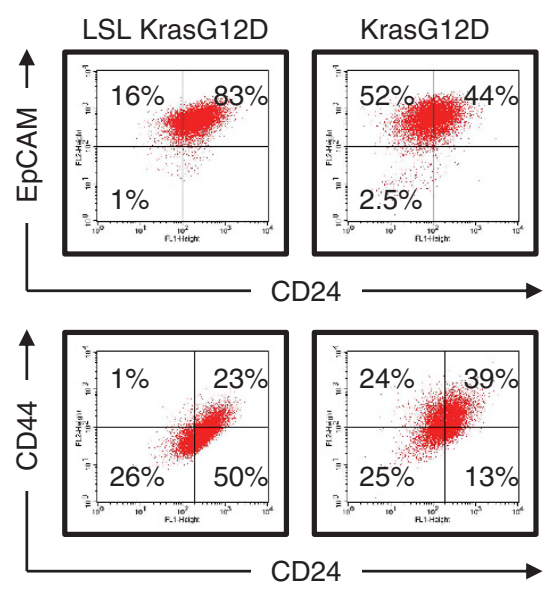

d

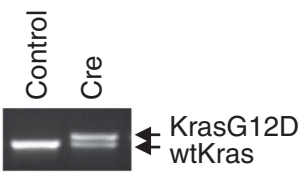

SCC

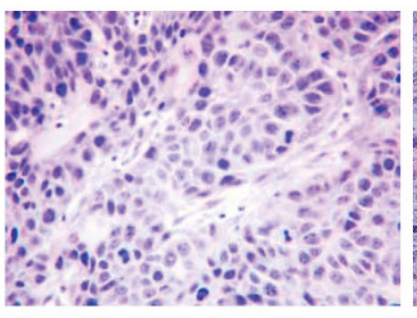

Sarcomatoid carcinoma
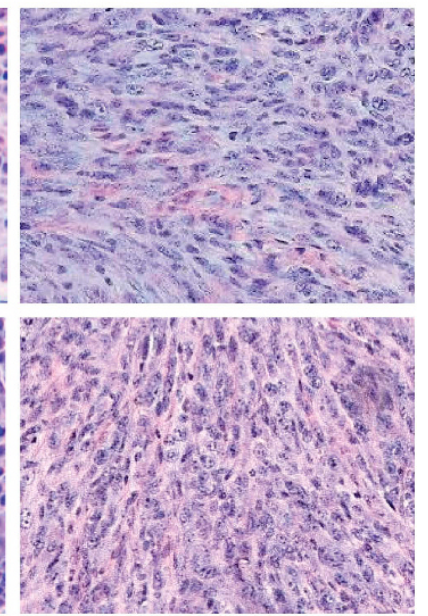

Figure 1 Identification of tumorigenic cells in KrasG12D-induced lung carcinoma. (a) FACS analysis of the purified LSL KrasG12D p53 ${ }^{\mathrm{KO}}$ and KrasG12D p53 ${ }^{\mathrm{KO}}$ lung epithelial cells using the indicated antibodies. (b, c) Western blot analysis of KrasG12D expression, (b) or pull-down of GTP-bound active Kras (c) in LSL KrasG12D p53 ${ }^{\mathrm{KO}}$ cells (inactive KrasG12D allele), KrasG12D p53 ${ }^{\mathrm{KO}}$ cells (active KrasG12D allele) or tumors derived from these cells. Passage numbers are indicated. MAPK is a loading control. (d) Genomic PCR of LSL KrasG12D p53 ${ }^{\mathrm{KO}}$ cells infected with Cre-expressing retroviruses distinguishes recombined KrasG12D allele from WT Kras allele by addition of $40 \mathrm{bp}$ in the intronic region. The nonrecombined LSL KrasG12D allele does not amplify under these conditions. (e) Representative images of tumors from mice injected with $\mathrm{CD} 34$ - and CD34 + KrasG12D p53 ${ }^{\mathrm{KO}}$ cells

development of $\mathrm{ADC}$ and SCC, we used three different approaches: (1) genetic or pharmacological inactivation of TGF-beta signaling in premalignant and cancer-derived cell lines; (2) dominant interference with the formation of SMAD complexes in these cell lines; and (3) constitutive activation of the TGF-beta receptor signaling in premalignant KrasG12D p53 ${ }^{\mathrm{KO}}$ cells and tumors.
We started by expressing a kinase-defective dominantnegative form of TBR2 (DN TBR2), which partially blocks TGF-beta pathway activity. Stable expression of DN TBR2 led to a shift of tumor morphology toward an exclusive squamous phenotype (Figure 3c). Remarkably, clonal cell lines that previously were able to give rise to two or three distinct tumor types now produced just one, that is, SCC (Figure 3c). 
a

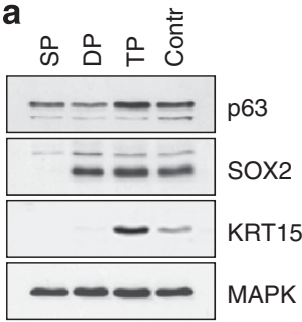

C

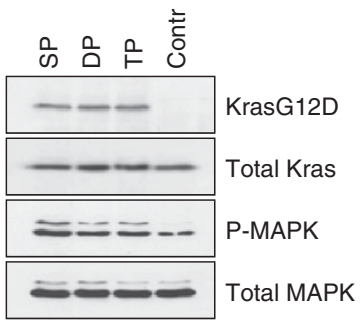

b

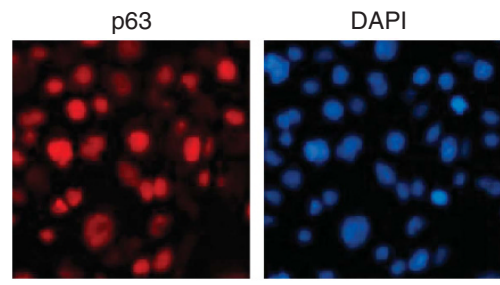

d

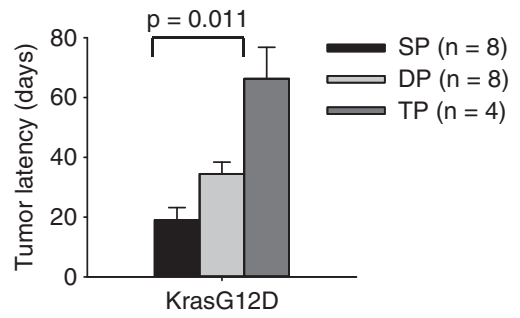

e
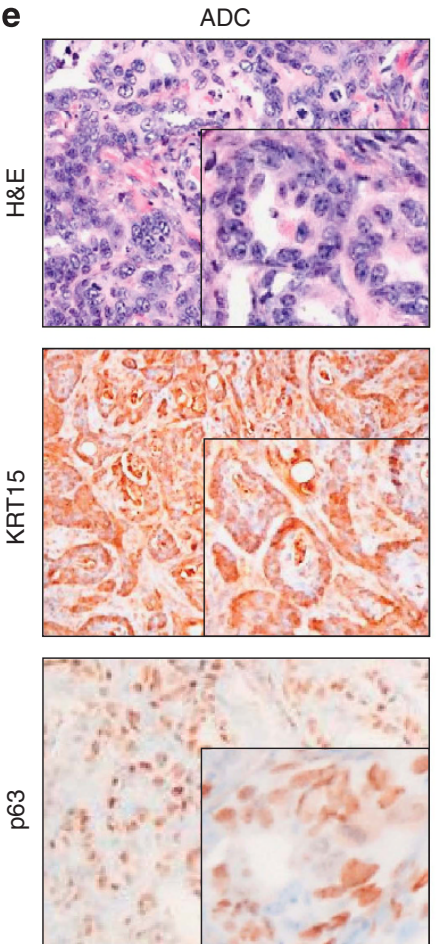

SCC
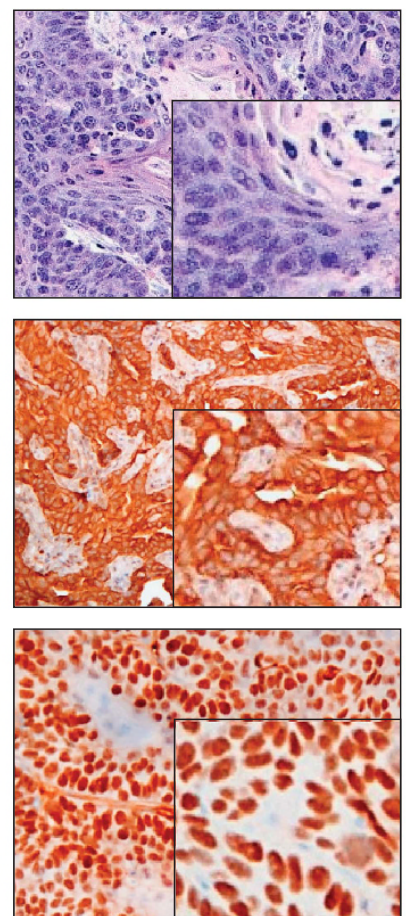

Sarcomatoid carcinoma
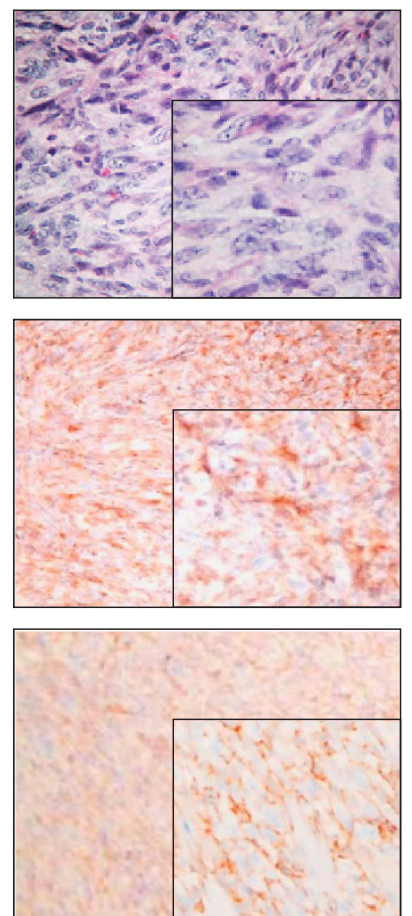

Figure 2 Cancers with distinct histological features originate from clonally derived cell populations. (a) Western blot analysis of LSL KrasG12D p53 ${ }^{\mathrm{KO}}$ cells and representative clonal derivatives of KrasG12D p53 ${ }^{\mathrm{KO}}$ cells. (b) Immunofluorescent staining of p63 protein in KrasG12D p5 ${ }^{\mathrm{KO}}$ cells. DAPI counterstain is shown. (c) Western blot analysis of Kras and P-MAPK expression in clonal KrasG12D p5 $3^{\mathrm{KO}}$ cells. (d) Tumor formation in nude mice by clonal KrasG12D p53 $3^{\mathrm{KO}}$ cells $\left(2 \times 10^{4}\right.$ cells per injection site). (e) H\&E and immunohistochemical staining of tumors from mice injected with clonal KrasG12D p53 ${ }^{\mathrm{KO}}$ cells. Expression of keratin-15 and p63 in ADC, SCC and SC is shown

No ADCs were detected in this group. Next, we tested the biological responses of clonal KrasG12D p53 ${ }^{\mathrm{KO}}$ cells to stable expression of a constitutively active TGF-beta receptor 1 (TBR1 T202D), which bypasses the TGF-beta and TBR2 requirement. Tumors derived from such cells were classified as either SCs or ADCs on the basis of predominant cell morphology (Figure 3d), whereas SCCs were no longer detected in this group. Western blot analysis of the transduced cell lines revealed that only moderate expression of the exogenous TBR1 T202D was achieved, but this was sufficient to generate increased chronic activation of TGF-beta signaling, as evidenced by SMAD2 phosphorylation (Figure 3d). These data indicate that repression of the TGF-beta pathway at the receptor level promotes the formation of SCC, whereas its constitutive activation promotes the formation of ADC and/ or SC. Most importantly, these data suggest that KrasG12D 
a

$\tan \frac{\infty}{2} \frac{\mathrm{N}}{\mathrm{a}}$

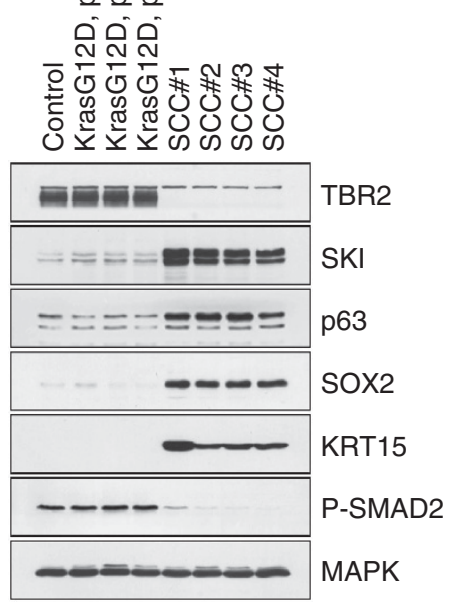

b

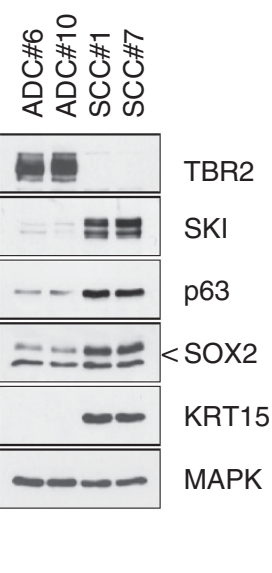

DNTBR2-transduced DP cells

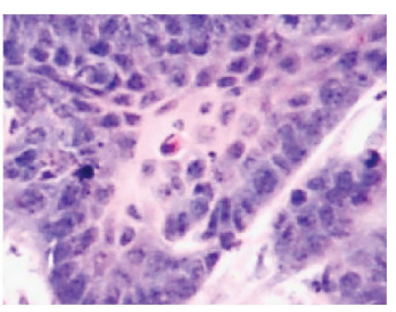

TBR1*-transduced DP cells

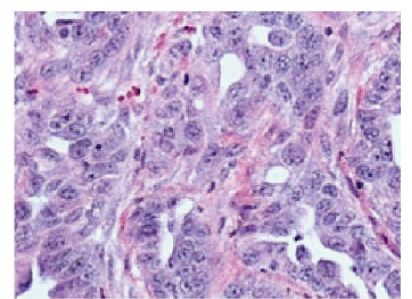

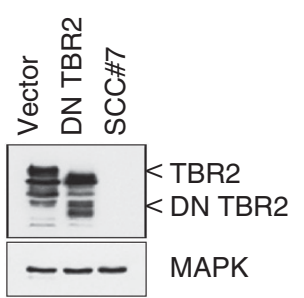

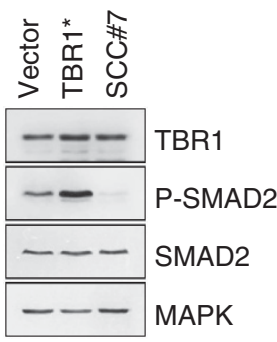

Figure 3 TGF-beta-mediated response regulates lineage commitment of lung cancer cells. (a, b) Western blot analysis of LSL KrasG12D p53 ${ }^{\mathrm{KO}}$ lung epithelial cells (control), premalignant and tumor-derived KrasG12D p53 ${ }^{\mathrm{KO}}$ cells. Passage numbers are indicated. (c) The histology of tumors derived from clonal KrasG12D p53 ${ }^{\mathrm{KO}}$ cells transduced with DN TBR2 mutant. Inset shows western blotting of DN TBR2 expression in the transduced cells. (d) The histology of tumors derived from clonal KrasG12D p53 ${ }^{\mathrm{KO}}$ cells transduced with TBR1 T202D mutant. Inset shows western blotting of TBR1 expression and SMAD2 phosphorylation in the transduced cells before injection

p5 $3^{\mathrm{KO}}$ cells are interconvertible for the tumor types they form (i.e., ADC, SC or SCC), and their differentiation potential is determined by TGF-beta-mediated signals.

Interference with the formation of active SMAD complexes promotes squamous cell carcinogenesis. We next sought to determine whether inactivation of SMAD signaling was of functional importance in lung carcinogenesis. Because the oncoprotein SKI acts as a potent negative regulator of the formation of active SMAD2/3SMAD4 complexes, ${ }^{29,30}$ we tested the consequences of expressing the SKI protein in various cell types. We found that ectopic expression of SKI in premalignant KrasG12D p5 ${ }^{\mathrm{KO}}$ cells, their clonal derivatives or cancer-derived ADC cell lines in all cases led to the subsequent development of carcinomas exhibiting pure squamous morphology (Figure 4a and Supplementary Figure 6). Moreover, these SKI-modified cancer cells acquired increased expression of p63, soX2 and KRT15 (Figure 4b), demonstrating functional loss of TGF-beta responsiveness in these cancer cells. Treatment of premalignant KrasG12D p53 ${ }^{\mathrm{KO}}$ cells or ADC-derived cell lines (e.g., ADC\#6, Figure 3b) with the TBR1 inhibitor SB431542 also induced robust expression of markers found in SCC, namely SKI, SOX2 and KRT15 (Figures 4c and d). We noted that SKI-expressing cells gave rise to tumors with an increased penetrance and shorter latency compared with cells transduced with vector alone or DN TBR2-expressing retroviruses (Figure $4 \mathrm{e}$ and Supplementary Figure). In contrast to the reduced tumor latency, there was no change in the rate of tumor growth in mice (Figure $4 \mathrm{f}$ and Supplementary Figure 7), suggesting that SKI does not alter cell growth per se, but may have an active role in maintaining tumorigenic properties of cancer cells. To test this idea, we serially transplanted SKI-expressing cancer cells into 
a

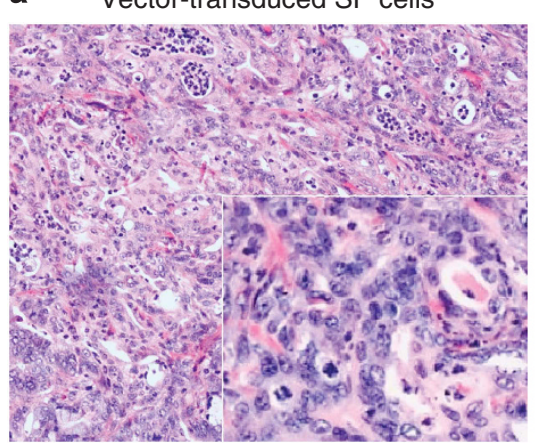

SKI-transduced SP cells

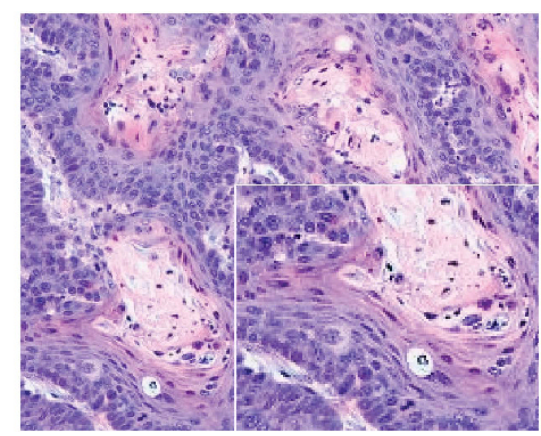

b

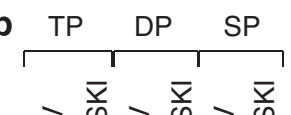

$>\overline{\frac{\Sigma}{\omega}}>\overline{\mathrm{s}}>\overline{\mathrm{s}}$

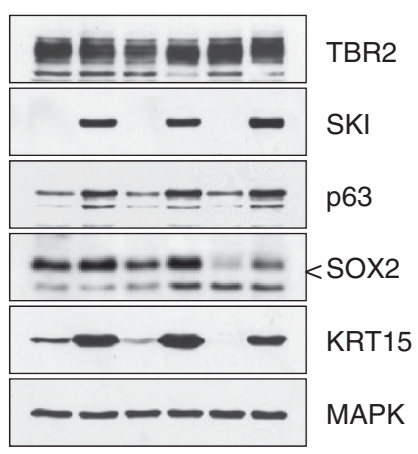

e

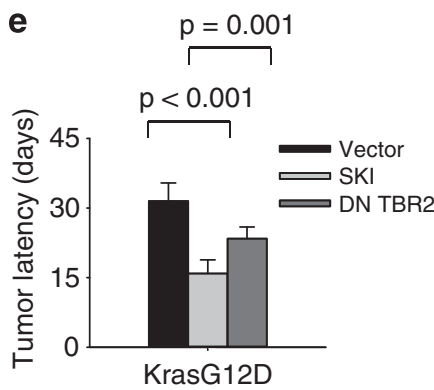

c

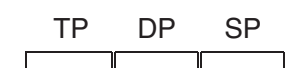

言品言品

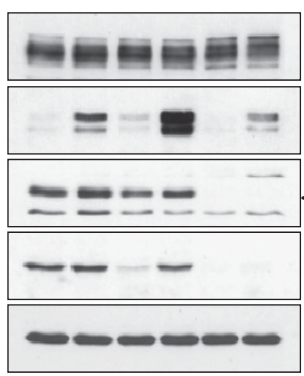

f

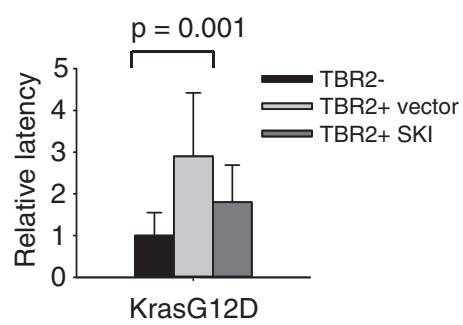

d

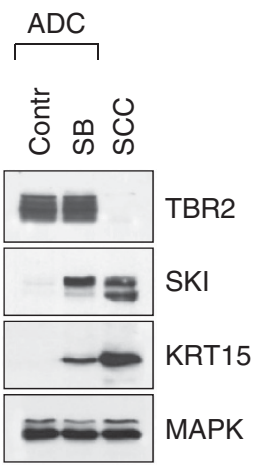

g

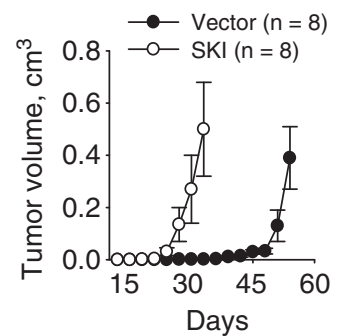

Figure 4 Interference with the formation of active SMAD complexes promotes squamous cell carcinogenesis. (a) Ectopic expression of SKI in clonal KrasG12D p53 ${ }^{\mathrm{KO}}$ cells (left panel) induces a shift toward squamous morphology (right panel). (b) Western blot analysis of clonal KrasG12D p53 ${ }^{\mathrm{KO}}$ cells transduced with vector alone or SKIexpressing retroviruses. (c, d) Western blot analysis of clonal premalignant KrasG12D p53 ${ }^{\mathrm{KO}}$ cells $(\mathbf{c})$, or primary ADC\#6 cells (d) incubated for 2 days in the presence of $1 \mu \mathrm{M}$ TGF-beta receptor inhibitor SB431542. (e) Ectopic expression of SKI or DN TBR2 in premalignant KrasG12D p53 ${ }^{\mathrm{KO}}$ cells accelerates tumorigenesis in nude mice $\left(2 \times 10^{4}\right.$ cells per injection site). (f) Ectopic expression of SKI in TBR2-positive ADC accelerates tumorigenesis in vivo. Latencies of tumor development were compared with TBR2negative SCC control. (g) Kinetics of tumor growth in nude mice injected with vector- or SKI-transduced KrasG12D p53 ${ }^{\mathrm{KO}}$ lung epithelial cells $\left(2 \times 10^{4}\right.$ cells per injection site)

secondary recipient mice. The secondary tumors arising from such transplanted cells indeed had shorter latency periods than the respective primary tumors, reflecting their increased tumor-initiating ability, and in all cases displayed the typical histological appearance of SCC (Figure $4 \mathrm{~g}$ and Supplementary Figure 7). The inverse relationship between TBR2 and SKI expression was also observed in human NSCLC cell lines (Supplementary Figure 7). Furthermore, immunostaining of a human lung carcinoma tissue array indicated that SKI is frequently expressed in SCC (Supplementary Figure 8), supporting its role in lung carcinogenesis.
SKI and SOX2 display partly redundant roles in lung carcinogenesis. Amplification of the SOX2 locus is a common event in lung SCCs. ${ }^{26,27}$ Given the importance of SOX2 in the maintenance of stem cell phenotypes and the apparent relationship between SOX2 expression status and tumor malignancy, ${ }^{5,31,32}$ we sought to establish the role of TGF-beta in the regulation of SOX2 expression. Notably, SOX2 has been reported to co-occupy SMAD4-binding loci in ES cells, ${ }^{33}$ and to antagonize TGF-beta's activity during reprogramming of mouse embryonic fibroblasts (MEFs) into an ES-like state. ${ }^{34,35}$ Therefore, we examined the specificity and reciprocity of TBR2, SKI and SOX2 expression in 
premalignant cells and the tumor-derived cell lines. We found that ectopic SOX2 caused stabilization of the SKI protein level in several cell types, including MEFs (Figure 5a), untransformed LSL KrasG12D p53 ${ }^{\mathrm{KO}}$ lung epithelial cells (LECs) (Figure 5a) and premalignant KrasG12D p53 ${ }^{\mathrm{KO}}$ cells (Figure 5a), but not in TBR2-negative cancer cell lines, implying that SOX2 overexpression causes a loss of TGF-beta responsiveness in cancer cells. Consistent with this notion, tumors generated from SOX2-transduced precancerous KrasG12D p53 ${ }^{\mathrm{KO}}$ cell lines or cancer-derived ADC cell lines (e.g., ADC\#6, Figure 3b) were structurally similar to the SCC tumors generated by DN TBR2- or SKIoverexpressing cells, exhibiting a characteristic squamous morphology with pronounced keratinization and formation of keratin pearls (Figure $5 b$ ). Thus, in addition to revealing a positive reciprocal relationship between SOX2 and SKI (Figure 5c), these data indicate that SOX2 and SKI may act in a partially redundant manner to promote a squamous phenotype of lung cancer cells.

Identification of KrasG12D expression signature in lung cancer cells. To further explore the role of TGF-beta signaling in lung tumorigenesis, we performed microarray analysis of isogenic ADC and SCC cell lines expressing endogenous KrasG12D (Figure 6a). Inoculated into nude mice, these cell lines produce ADCs and squamous a

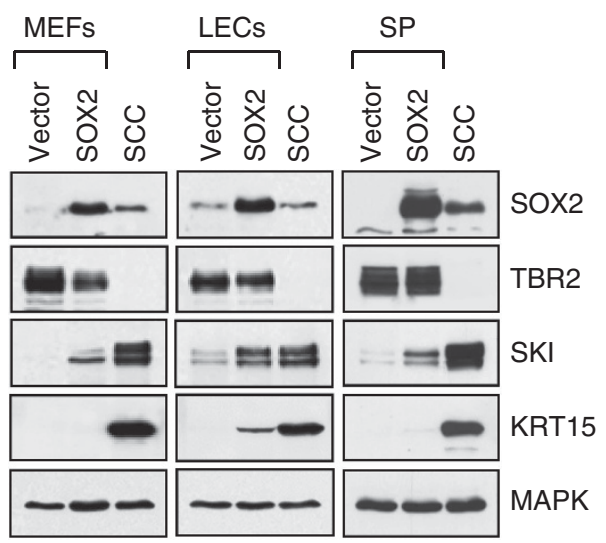

C

$\mathrm{EpCAM}+\mathrm{CD} 104+\mathrm{CD} 49 \mathrm{f}+$ CD44+ CD24lo SCA1+<smiles></smiles>

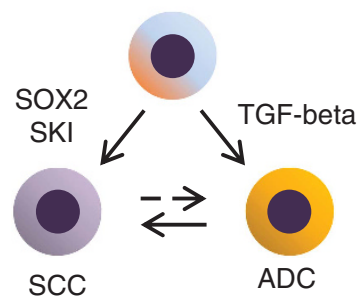

b Vector-transduced SP cells

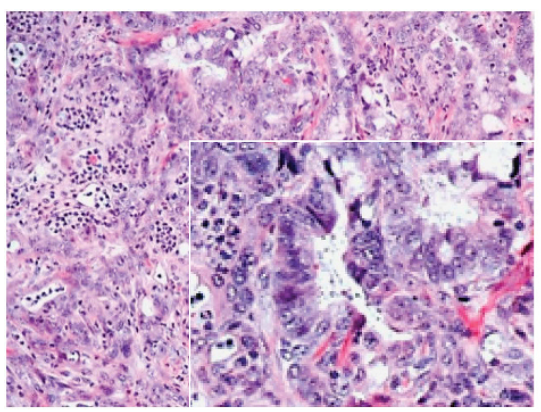

Vector-transduced ADC

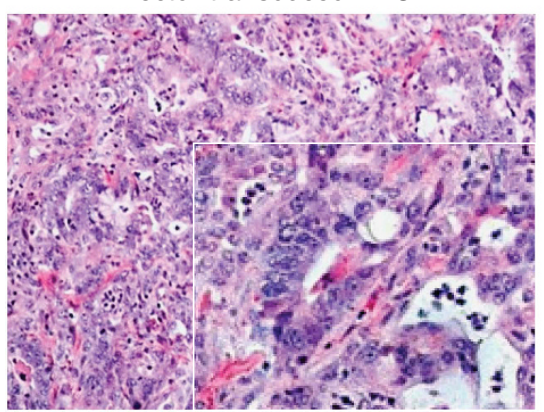

SOX2-transduced SP cells

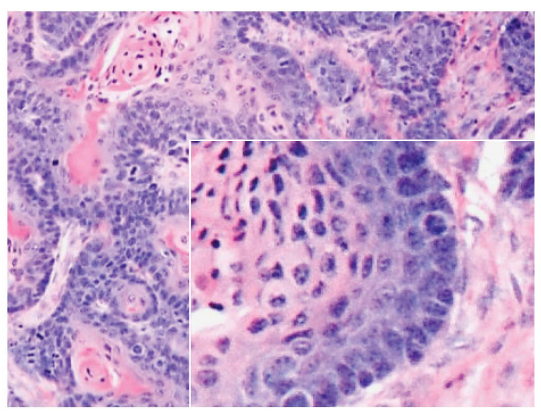

SOX2-transduced ADC

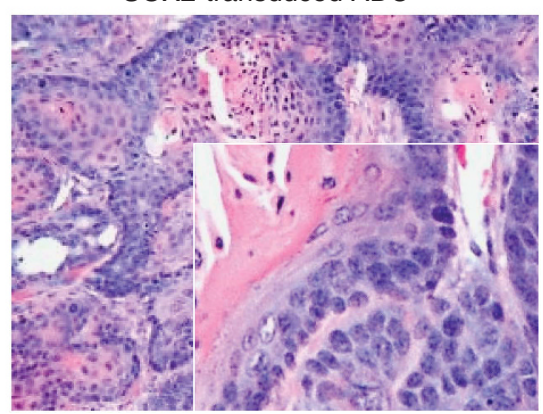

Figure 5 SOX2 and SKI display partly redundant roles in squamous cell carcinogenesis. (a) Western blot analysis of SOX2-transduced primary fibroblasts (MEFs), LSL KrasG12D p53 ${ }^{\mathrm{KO}}$ lung epithelial cells (LECs) and clonal KrasG12D p53 ${ }^{\mathrm{KO}}$ cells. SCC cell line \#7 is shown as control. (b) The histology of tumors derived from vectortransduced (left) or SOX2-transduced (right) KrasG12D p53 ${ }^{\mathrm{KO}}$ cells or ADC\#6 cell line. (c) SOX2 and SKI display reciprocal and partly redundant roles in the regulation of plasticity and lineage commitment of lung cancer cells. Note that SP cells are predisposed to the development of ADC, whereas TP cells are predisposed to the development of SCC 
carcinomas, respectively. The SCC was generated from the parental ADC by means of SKI overexpression (see Figure $4 a)$. We identified $\sim 530$ genes differentially expressed by more than four-fold in ADC and $\sim 790$ genes differentially expressed in SCC compared with untransformed controls (Figures $6 b$ and $c$ ). Many of the gene expression changes were shared between ADC and SCC and also have been found in human cancers (Supplementary Table 1). Besides Sox2, p63 and Krt15, the known differentially expressed genes in each cancer type represented several functional groups, including metabolism, cell adhesion and migration, signal transduction and transcriptional regulation (Supplementary Table 1). Of the four previously identified gene expression signatures predictive of cytogenetic and molecular subtypes of human lung SCC (i.e., primitive, basal, secretory and classical), ${ }^{5,36}$ SKIinduced tumors closely resembled the primitive subtype (Supplementary Table 2). Although an active MYC expression module is observed in many cancer types, ${ }^{37}$ a set of genes reported to be induced by MYC activation showed no significant overlap with our data sets (Figure 6d). By contrast, the activity of TGF-beta/SMAD expression module (genes regulated via the canonical TGF-beta pathway) was markedly repressed in SKI-expressing tumor cells (Figure 6d), the core pluripotency module (target genes of SOX2 and other pluripotency-associated transcription factors) was activated in SCC cells (Figure 6d), whereas the NF-kappaB gene- expression module was activated in ADC cells (Figure 6d). Western blotting confirmed that SKI is often coexpressed with SOX2, OCT4 and KLF4 (Supplementary Figure 7). Considering the fact that, pathologically, lung ADCs carrying Kras and p53 mutations are classified as poorly differentiated, high-grade cancers, ${ }^{38,39}$ the expression of pluripotency-associated transcription factors indicates that KrasG12D-generated SCCs are composed of cells that are relatively immature.

\section{Discussion}

The main objective of this study was to understand the role of TGF-beta signaling in epithelial plasticity and lung carcinogenesis. Toward this goal, we developed isolation and culture conditions that favor the growth of lung epithelial cells with stem cell-like properties. We identified a population of cells in the adult lung that bear the EpCAM + CD104 + CD49f + CD44+CD24loSCA1 + phenotype and can be clonally expanded in culture. On the basis of their FACS profile, our cells bear close phenotypic resemblance to the recently described population of self-renewing AECs, ${ }^{19,20}$ which express little or no $\mathrm{CC} 10$ or $\mathrm{SpC}$, but give rise to the differentiated airway and alveolar cells both ex vivo and in vivo. ${ }^{19,20}$ We find that these cells represent a heterogeneous population that can be discriminated on the basis of p63, SOX2 and KRT15 expression, consistent with the notion

a
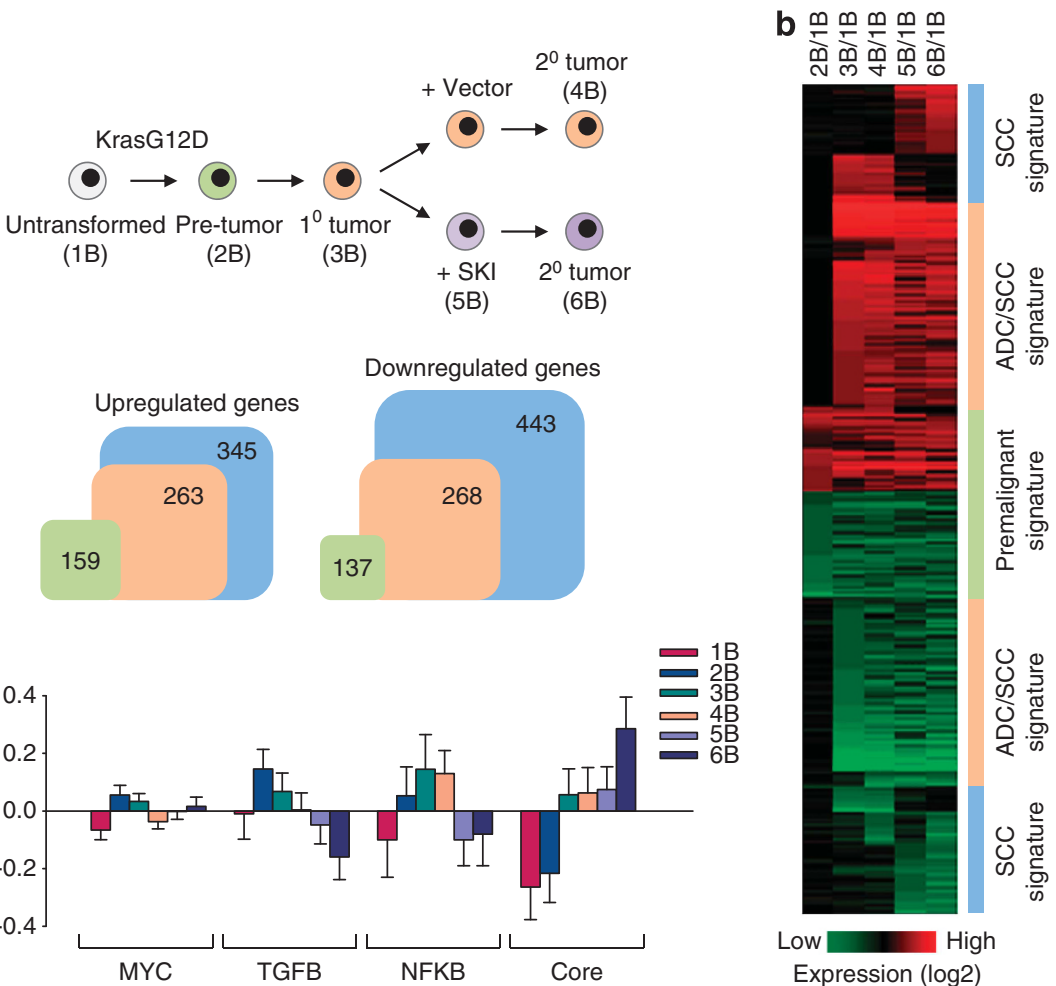

Figure 6 Identification of a KrasG12Di expression signature in lung cancer cells. (a) Schematic of the experimental approach for microarray analysis of untransformed $(1 \mathrm{~B})$, premalignant $(2 \mathrm{~B})$ and tumor-derived KrasG12D p53 ${ }^{\mathrm{KO}}$ cells $(3 \mathrm{~B}-6 \mathrm{~B})$. Primary ADC\#6 (3B) was used to derive the corresponding secondary tumors (4B and 6B). (b) Heat maps showing differentially expressed genes compared with untransformed controls (1B). Genes with four-fold or greater difference are shown. (c) The number of differentially expressed genes in premalignant KrasG12D p53 ${ }^{\mathrm{KO}}$ cells (green), ADC (orange) and SCC (blue) is shown. (d) Activity of gene regulatory modules in untransformed, premalignant and tumor-derived KrasG12D p53 ${ }^{\mathrm{KO}}$ cells 
that cancer cells rely on the continuous activation of lineagedetermining transcription factors involved in cell-fate decisions and lineage commitment. ${ }^{40}$ This in turn implies that the cell(s) of origin of lung cancer are more diverse than previously recognized. ${ }^{12,14,16}$

The two most common histological types of human lung cancer, ADC and SCC, are sufficiently distinct to be recognized as separate entities with different mutational profiles and pathological characteristics. ${ }^{7,41}$ Whereas ADCs tend to arise peripherally and are believed to develop from alveolar or bronchiolar cells, SCCs, particularly those that arise centrally, evolve from preinvasive lesions termed bronchial dysplasia, which then progress to carcinoma in situ and invasive carcinoma. ${ }^{16,42}$ The most notable difference between the two main types of human NSCLC is that EGFR and Kras mutations are more common in ADCs (about 30-50\%) than in SCCs (5-7\%). A recent comprehensive genomic characterization of human lung SCCs from large cohorts of patients revealed that the vast majority of tumors ( $\sim 70 \%$ ) carry genetic alterations in at least one of the three overlapping cellular pathways: receptor tyrosine kinases (RTK), PI3K/AKT and RAS. ${ }^{5}$ Thus, gene expression and pathway signatures of lung ADC and SCC show both similarities and differences.

A striking finding of this study is that a population of lungderived cells with the immature epithelial phenotype of precursor cells is able to generate several different histological types of cancer, including ADC and SCC, rather than being restricted to one type. Our data indicate that, in the context of mutant Kras-driven tumors, repression of the TGFbeta pathway strongly promotes the formation of SCC, whereas activators of this pathway favor the development of ADC. This was true for premalignant cells and even for tumor cell lines derived from primary ADCs, which, upon disruption of TGF-beta signaling, subsequently gave rise to SSCs. We went on to demonstrate a close connection between TGFbeta signaling and SOX2 expression, and identify SKI and SOX2 as regulators of lineage commitment of KrasG12Dexpressing lung cancer cells. Our present observations provide support to the view that the biological state of the cell of origin (i.e., the cellular context) is an important determinant of carcinogenic potential. ${ }^{43-45}$ Questions remain as to how one transformed cell type turns into a different cell type. Our evidence indicates that this process bears the characteristics of lineage reprogramming, a type of metaplasia driven by fate-determining transcription factors (e.g., SOX2), wherein cells switch lineages to create another cell type without induction of hybrid cell phenotypes. ${ }^{46}$ In agreement with our results, recent studies indicate that the startling phenotypic heterogeneity of cells within tumors can be driven by reversible epigenetic changes within populations of tumorigenic and nontumorigenic cancer cells. ${ }^{43,44,47,48}$ Our study extends the concept of cellular plasticity in cancer by showing that KrasG12D-transformed lung epithelial cells acquire the ability to give rise to several different tumor types rather than one type. As such, our findings represent a profound divergence from the accepted hierarchical models of epithelial carcinogenesis and may have widespread implications to various types of malignancies. On a practical note, they may explain why some mouse lung ADC models also develop squamous morphology. ${ }^{49}$ Although some caution is warranted in extrapolating these results to the human disease insofar as they are not yet corroborated by other evidence, our results establish a new paradigm for our understanding of pathological implications of cellular plasticity in cancer and, more specifically, its role in lung cancer causation and progression. Given the major differences between human and mouse airway cell populations, ${ }^{16,18,24}$ and taking into consideration the role of carcinogens in human lung cancer that are lacking in this model, it is important to establish whether there are causal relationships between oncogenic mutations discovered in major types of lung cancer, target cells of transformation and the subtypes of lung cancer.

\section{Materials and Methods}

All animal studies were approved by the Institutional Animal Care and Use Committee at Stony Brook University. We used previously described LSL KrasG12D mice ${ }^{11}$ crossed to p53-null mice. Adult lung epithelial cells were prepared from 4- to 6-week-old mice. Lungs were washed in PBS, minced and treated with collagenase/ dispase (Roche, Indianapolis, IN, USA, final concentration $2 \mathrm{mg} / \mathrm{ml}$ ) for $2-3 \mathrm{~h}$. The digested tissue was washed in DMEM and filtered through a $40-\mu$ m nylon filter. Red blood cells (RBC) were lysed with RBC lysis solution $\left(0.15 \mathrm{M} \mathrm{NH}_{4} \mathrm{Cl}, 10 \mathrm{mM} \mathrm{KHCO}_{3}\right.$, $0.1 \mathrm{mM}$ EDTA) for $2 \mathrm{~min}$, followed by a washing step. All cells, unless otherwise specified, were grown on gelatinized plates in CnT-17 medium (CellnTec, Zen-Bio, Research Triangle, NC, USA). To delete the transcriptional termination cassette (Lox-Stop-Lox) containing the puromycin resistance gene and thus activate expression of the KrasG12D protein, we used a self-excising retroviral vector expressing Cre. ${ }^{23}$ The presence of the recombined KrasG12D allele in the cells was confirmed by selection of cells in puromycin. ${ }^{50}$ The efficiency of recombination was $\geq 90 \%$, as measured on days 4-8 post infection. PCR primers for detecting the WT and KrasG12D alleles were as follows: $5^{\prime}$-tccaacacagatgttcttaggctac and $5^{\prime}$-tccg aattcagtgactacagatgtacagag. PCR products were separated on a $2 \%$ agarose gel. Successful recombination (single LoxP site) yields an $\sim 340$-bp product ( $\sim 300$-bp in WT Kras allele). For flow cytometry, cells were lifted with Accutase (Sigma), stained with FITCl-, PE- or APC-conjugated antibodies to CD90 (Thy1.2), SCA1 (stem cell antigen 1), CD326 (EpCAM), CD104 (integrin $\beta 4$ ), CD49f (integrin $\alpha 6$ ), CD44, CD34 and CD24 (eBioscience or BD, San Diego, CA, USA) and analyzed using FACSCalibur (BD) with the CellQuest software. Cell viability was measured using propidium iodide $(\mathrm{PI})$ staining. Cells were injected subcutaneously into nude mice at 4 sites on the back of the mouse $\left(2 \times 10^{4}\right.$ cells per injection site). We defined tumor latencies as the period between injection of tumorigenic cells into mice and the appearance of tumors of $\geq 1 \mathrm{~mm}$ in diameter. The survival end point was a tumor diameter of $1 \mathrm{~cm}$. The xenograft tumors were passaged in vitro as cell lines to remove contaminating non-neoplastic cells, facilitating the detection of mutations. The cell lines were derived from TBR2-negative tumors (morphologically pure SCCs that expressed high levels of KRT15 and the transcription factors SKI, p63 and SOX2) and TBR2-positive tumors (either ADCs or SCs, which had low or no expression of KRT15, SKI, p63 or SOX2). The lines are referenced based on the type of tumor from which they were derived, for example, SCC\#1. When serially transplanted into secondary recipient mice, the secondary tumors in all cases displayed the histological appearance of the respective primary tumors. For sphereforming assays, single-cell suspensions were cultured in ultralow attachment 6-well plates (Corning, Tewksbury, MA, USA) with CnT-17 medium in the presence or absence of exogenous TGF-beta, EGF and bFGF $\left(10 \mathrm{ng} / \mathrm{ml}\right.$ each) at $2 \times 10^{4}-10^{5}$ cells per well. Standard protocols were followed for western blot analysis. We used antibodies specific for Kras (Ab-1), Ras Asp12 (Ab-1), Pan-Ras (F132-62, all from Millipore, Billerica, MA, USA), Hras (6100001, BD), MYC (N-262), SKI (H-329, both from Santa Cruz), SOX2 (AB5603, Millipore), KRT15 (AJ1220b, Abgent, San Diego, CA, USA), KRT17/19 (D32D9, Cell Signaling) and MAPK (05-157, Upstate). The Ras activation assay was performed using an agarose-bound GST-fused Rasbinding domain (RBD) of Raf-1 (Cell Biolabs, San Diego, CA, USA). Briefly, cells were plated at equal density, grown in $\mathrm{CnT}-17$ medium, washed with cold PBS and lysed. The activated Ras was pulled down with agarose-conjugated Raf-1 RBD, followed by SDS/PAGE gel electrophoresis and immunoblotting with anti-Kras, Hras and Pan-Ras antibodies. We used replication-defective retroviral vectors encoding 
MYC, OCT4, SOX2, SKI, TBR2, dominant-negative TBR2 mutant (amino acids 1-283 of human TBR2) and constitutively active TBR1 (TBR1 T202D).

Expression analysis. Lung Disease Spectrum Tissue arrays (BC041115a) were obtained from US Biomax (Rockville, MD, USA). For gene expression microarrays, total cellular RNA was extracted using the RNeasy Mini Kit (Qiagen, Germantown, MD, USA). One-color hybridizations of labeled cRNAs (2 technical replicas) were performed against the mouse llumina MouseRef-8 Expression BeadChip $25 \mathrm{~K}$ microarray at Roswell Park Cancer Institute (Buffalo, NY, USA). Microarray signals were processed with GenomeStudio Gene Expression Module (GSGX) Version 1.6.0 (Illumina, San Diego, IL, USA). Differentially expressed genes were defined based on $>2$ - or 4-fold change. Data were background corrected and quantile normalized. A small offset was applied to bring values above 0 . The heat maps were generated by calculating ratios of expression in each sample versus control. The log2 values were then supplied to the heat-map function of the R statistical package. Module expression analysis was conducted as described. ${ }^{37}$ Average gene expression values (log2) of all genes were set as baseline 0 . The gene expression values $(\log 2)$ of each module relative to the overall average were represented as mean \pm S.E.M. We used NFkB, PRC1, PRC2 and TGF-beta modules defined by The Broad Institute repository of modules (http://www.broadinstitute.org/gsea).The MYC module comprises 500 targets of MYC, MAX, N-MYC, DMAP1, E2F1, E2F4 and ZFX; the Core module includes previously known factors in core regulatory circuitry, such as NANOG, OCT4, REST, SOX2, TCF3 and REX1; and the PRC module is composed of 560 targets of PRC cluster proteins PHC1, RNF2, EED and SUZ12. ${ }^{37}$ Statistical analyses were performed using Student's $t$-test. $P \leq 0.05$ was considered statistically significant.

\section{Conflict of Interest}

The authors declare no conflict of interest.

Acknowledgements. This work was supported by US Public Health Service Grant CA42573 from the National Cancer Institute to MJH. We thank Alice Nemajerova, Sulan Xu, Ken Shroyer and Mallory Korman of the Department of Pathology (Stony Brook University) for their invaluable help in histologic and immunohistologic analysis of mouse and human lung tumor samples, and Dr. Raffaella Sordella (Cold Spring Harbor Labs) for providing human lung cancer cell lines.

\section{Author contributions}

$\mathrm{MH}$ and OP designed the research; II, JL and OP performed the research; II and OP contributed new reagents/analytic tools; and $\mathrm{MH}$ and $\mathrm{OP}$ analyzed the data and wrote the paper.

1. Karnoub AE, Weinberg RA. Ras oncogenes: split personalities. Nat Rev Mol Cell Biol2008; 9: $517-531$.

2. Pao W, Girard N. New driver mutations in non-small-cell lung cancer. Lancet Oncol 2011; 12: $175-180$.

3. Yatabe $\mathrm{Y}$, Borczuk AC, Powell CA. Do all lung adenocarcinomas follow a stepwise progression? Lung Cancer 2011; 74: 7-11.

4. MacConaill LE. Advancing personalized cancer medicine in lung cancer. Arch Pathol Lab Med 2012; 136: 1210-1216.

5. Cancer Genome Atlas Research Network. Comprehensive genomic characterization of squamous cell lung cancers. Nature 2012; 489: 519-525.

6. Ding L, Getz G, Wheeler DA, Mardis ER, McLellan MD, Cibulskis K et al. Somatic mutations affect key pathways in lung adenocarcinoma. Nature 2008; 455: 1069-1075.

7. Govindan R, Ding L, Griffith M, Subramanian J, Dees ND, Kanchi KL et al. Genomic landscape of non-small cell lung cancer in smokers and never-smokers. Cell 2012; 150 1121-1134

8. Imielinski M, Berger AH, Hammerman PS, Hernandez B, Pugh TJ, Hodis E et al. Mapping the hallmarks of lung adenocarcinoma with massively parallel sequencing. Cell 2012; 150 $1107-1120$

9. Kan Z, Jaiswal BS, Stinson J, Janakiraman V, Bhatt D, Stern HM et al. Diverse somatic mutation patterns and pathway alterations in human cancers. Nature 2010; 466: 869-873.

10. Guerra C, Mijimolle N, Dhawahir A, Dubus P, Barradas M, Serrano M et al. Tumor induction by an endogenous K-ras oncogene is highly dependent on cellular context. Cancer Cell 2003; 4: 111-120.
11. Tuveson DA, Shaw AT, Willis NA, Silver DP, Jackson EL, Chang S et al. Endogenous oncogenic K-ras(G12D) stimulates proliferation and widespread neoplastic and developmental defects. Cancer Cell 2004; 5: 375-387.

12. Kim CF, Jackson EL, Woolfenden AE, Lawrence S, Babar I, Vogel S et al. Identification of bronchioalveolar stem cells in normal lung and lung cancer. Cell 2005; 121: 823-835

13. Liu C, Sage JC, Miller MR, Verhaak RG, Hippenmeyer S, Vogel H et al. Mosaic analysis with double markers reveals tumor cell of origin in glioma. Cell 2011; 146: 209-221.

14. Xu X, Rock JR, Lu Y, Futtner C, Schwab B, Guinney J et al. Evidence for type II cells as cells of origin of K-Ras-induced distal lung adenocarcinoma. Proc Natl Acad Sci USA 2012; 109: 4910-4915.

15. Rawlins EL, Okubo T, Xue Y, Brass DM, Auten RL, Hasegawa $\mathrm{H}$ et al. The role of Scgb1a1 + Clara cells in the long-term maintenance and repair of lung airway, but not alveolar, epithelium. Cell Stem Cell 2009; 4: 525-534.

16. Rock JR, Hogan BL. Epithelial progenitor cells in lung development, maintenance, repair, and disease. Annu Rev Cell Dev Biol 2011; 27: 493-512.

17. Lin C, Song H, Huang C, Yao E, Gacayan R, Xu SM et al. Alveolar type II cells possess the capability of initiating lung tumor development. PloS One 2012; 7: e53817.

18. Kumar PA, Hu Y, Yamamoto Y, Hoe NB, Wei TS, Mu D et al. Distal airway stem cells yield alveoli in vitro and during lung regeneration following H1N1 influenza infection. Cell 2011; 147: 525-538.

19. Chapman HA, Li X, Alexander JP, Brumwell A, Lorizio W, Tan K et al. Integrin alpha6beta4 identifies an adult distal lung epithelial population with regenerative potential in mice. J Clin Invest 2011; 121: 2855-2862.

20. McQualter JL, Yuen K, Williams B, Bertoncello I. Evidence of an epithelial stem/progenitor cell hierarchy in the adult mouse lung. Proc Natl Acad Sci USA 2010; 107: 1414-1419.

21. Rock JR, Barkauskas CE, Cronce MJ, Xue Y, Harris JR, Liang J et al. Multiple stromal populations contribute to pulmonary fibrosis without evidence for epithelial to mesenchymal transition. Proc Natl Acad Sci USA 2011; 108: E1475-E1483.

22. Slack JM. Metaplasia and transdifferentiation: from pure biology to the clinic. Nat Rev Mol Cell Biol 2007; 8: 369-378

23. Silver DP, Livingston DM. Self-excising retroviral vectors encoding the Cre recombinase overcome Cre-mediated cellular toxicity. Mol Cell 2001; 8: 233-243.

24. Cole BB, Smith RW, Jenkins KM, Graham BB, Reynolds PR, Reynolds SD. Tracheal basal cells: a facultative progenitor cell pool. Am J Pathol 2010; 177: 362-376.

25. Gontan C, de Munck A, Vermeij M, Grosveld F, Tibboel D, Rottier R. Sox2 is important for two crucial processes in lung development: branching morphogenesis and epithelial cell differentiation. Dev Biol 2008; 317: 296-309.

26. Bass AJ, Watanabe H, Mermel CH, Yu S, Perner S, Verhaak RG et al. SOX2 is an amplified lineage-survival oncogene in lung and esophageal squamous cell carcinomas. Nat Genet 2009; 41: 1238-1242.

27. Hussenet T, Dali S, Exinger J, Monga B, Jost B, Dembele D et al. SOX2 is an oncogene activated by recurrent $3 \mathrm{q} 26.3$ amplifications in human lung squamous cell carcinomas. PloS One 2010; 5: e8960

28. Sanchez-Palencia A, Gomez-Morales M, Gomez-Capilla JA, Pedraza V, Boyero L, Rosell $\mathrm{R}$ et al. Gene expression profiling reveals novel biomarkers in nonsmall cell lung cancer. Int J Cancer 2011; 129: 355-364.

29. Luo K, Stroschein SL, Wang W, Chen D, Martens E, Zhou S et al. The Ski oncoprotein interacts with the Smad proteins to repress TGFbeta signaling. Genes Dev 1999; 13: 2196-2206.

30. Sun Y, Liu X, Ng-Eaton E, Lodish HF, Weinberg RA. SnoN and Ski protooncoproteins are rapidly degraded in response to transforming growth factor beta signaling. Proc Natl Acad Sci U SA 1999; 96: 12442-12447.

31. Rudin CM, Durinck S, Stawiski EW, Poirier JT, Modrusan Z, Shames DS et al. Comprehensive genomic analysis identifies SOX2 as a frequently amplified gene in small-cell lung cancer. Nat Genet 2012; 44: 1111-1116.

32. Arnold K, Sarkar A, Yram MA, Polo JM, Bronson R, Sengupta S et al. Sox2( + ) adult stem and progenitor cells are important for tissue regeneration and survival of mice. Cell Stem Cell 2011; 9: 317-329.

33. Fei T, Xia K, Li Z, Zhou B, Zhu S, Chen H et al. Genome-wide mapping of SMAD target genes reveals the role of BMP signaling in embryonic stem cell fate determination. Genome Res 2010; 20: 36-44.

34. Ichida JK, Blanchard J, Lam K, Son EY, Chung JE, Egli D et al. A small-molecule inhibitor of tgf-Beta signaling replaces sox2 in reprogramming by inducing nanog. Cell Stem Cell 2009; 5: 491-503.

35. Maherali N, Hochedlinger K. Tgfbeta signal inhibition cooperates in the induction of iPSCs and replaces Sox2 and cMyc. Curr Biol 2009; 19: 1718-1723.

36. Wilkerson MD, Yin X, Hoadley KA, Liu Y, Hayward MC, Cabanski CR et al. Lung squamous cell carcinoma mRNA expression subtypes are reproducible, clinically important, and correspond to normal cell types. Clin Cancer Res 2010; 16: 4864-4875.

37. Kim J, Woo AJ, Chu J, Snow JW, Fujiwara Y, Kim CG et al. A Myc network accounts for similarities between embryonic stem and cancer cell transcription programs. Cell 2010; 143: 313-324.

38. Hayes DN, Monti S, Parmigiani G, Gilks CB, Naoki K, Bhattacharjee A et al. Gene expression profiling reveals reproducible human lung adenocarcinoma subtypes in multiple independent patient cohorts. J Clin Oncol 2006; 24: 5079-5090. 
39. West L, Vidwans SJ, Campbell NP, Shrager J, Simon GR, Bueno R et al. A novel classification of lung cancer into molecular subtypes. PloS One 2012; 7: e31906.

40. Sarkar A, Hochedlinger $K$. The sox family of transcription factors: versatile regulators of stem and progenitor cell fate. Cell Stem Cell 2013; 12: 15-30.

41. Janku F, Stewart DJ, Kurzrock R. Targeted therapy in non-small-cell lung cancer-is it becoming a reality? Nat Rev Clin Oncol 2010; 7: 401-414.

42. Neuringer IP, Randell SH. Stem cells and repair of lung injuries. Respir Res 2004; $5: 6$.

43. Chaffer CL, Brueckmann I, Scheel C, Kaestli AJ, Wiggins PA, Rodrigues LO et al. Normal and neoplastic nonstem cells can spontaneously convert to a stem-like state. Proc Natl Acad Sci USA 2011; 108: 7950-7955.

44. Quintana E, Shackleton M, Foster HR, Fullen DR, Sabel MS, Johnson TM et al. Phenotypic heterogeneity among tumorigenic melanoma cells from patients that is reversible and not hierarchically organized. Cancer Cell 2010; 18: 510-523.
45. Schober M, Fuchs E. Tumor-initiating stem cells of squamous cell carcinomas and their control by TGF-beta and integrin/focal adhesion kinase (FAK) signaling. Proc Natl Acad Sci USA 2011; 108: 10544-10549.

46. Vierbuchen $T$, Wernig M. Direct lineage conversions: unnatural but useful? Nat Biotechnol 2011; 29: 892-907.

47. Gupta PB, Fillmore CM, Jiang G, Shapira SD, Tao K, Kuperwasser C et al. Stochastic state transitions give rise to phenotypic equilibrium in populations of cancer cells. Cell 2011; 146: 633-644.

48. Schwitalla S, Fingerle AA, Cammareri P, Nebelsiek T, Goktuna SI, Ziegler PK et al. Intestinal tumorigenesis initiated by dedifferentiation and acquisition of stem-cell-like properties. Cell 2013; 152: 25-38.

49. Farago AF, Snyder EL, Jacks T. SnapShot: lung cancer models. Cell 2012; 149: 246-246 e241.

50. Ischenko I, Zhi J, Moll UM, Nemajerova A, Petrenko O. Direct reprogramming by oncogenic Ras and Myc. Proc Natl Acad Sci USA 2013; 110: 3937-3942.

Supplementary Information accompanies this paper on Cell Death and Differentiation website (http://www.nature.com/cdd) 